\title{
CHARACTERIZATIONS OF WEAKLY CHAOTIC MAPS OF THE INTERVAL
}

\author{
V. V. FEDORENKO, A. N. SARKOVSKII, AND J. SMÍTAL
}

(Communicated by R. Daniel Mauldin)

\begin{abstract}
We prove, among others, the following relations between notions of chaos for continuous maps of the interval: (i) A map $f$ is not chaotic in the sense of $\mathrm{Li}$ and Yorke iff $f$ restricted to the set of its $\omega$-limit points is stable in the sense of Ljapunov. (ii) The topological entropy of $f$ is zero iff $f$ restricted to the set of chain recurrent points is not chaotic in the sense of $\mathrm{Li}$ and Yorke, and this is iff every trajectory is approximable by trajectories of periodic intervals.
\end{abstract}

\section{INTRODUCTION}

Let $I$ be a compact real interval, and $C(I, I)$ the space of continuous maps $I \rightarrow I$ with the uniform metric. For any $f \in C(I, I)$ and any nonnegative integer $n, f^{n}$ denotes the $n$th iterate of $f, h(f)$ the topological entropy of $f$, and for any $x \in I, \omega_{f}(x)$ is the $\omega$-limit set of $x$. A map $f$ is said to be chaotic (on a set $A \subseteq I$ ) if for some $x$ and $y$ from $A$,

$$
\begin{gathered}
\limsup _{n \rightarrow \infty}\left|f^{n}(x)-f^{n}(y)\right|>0, \\
\liminf _{n \rightarrow \infty}\left|f^{n}(x)-f^{n}(y)\right|=0 .
\end{gathered}
$$

This chaos is just the chaos in the sense of $\mathrm{Li}$ and Yorke [5]; other, equivalent definitions, including the original one, can be found in [12, 4]. Recall that the maps $f$ with $h(f)>0$ are chaotic. However, there are also chaotic maps with zero topological entropy $[6,12]$; they are referred to as weakly chaotic maps. In the sequel, we give relations between the above notions, and such notions as stability in the sense of Ljapunov or approximability of trajectories by trajectories of periodic points or intervals, that can be considered as kinds of nonchaotic behavior.

We use the following notation for standard subsets of $I: \operatorname{Per}(f), \operatorname{Rec}(f)=$ $\left\{x \in I ; x \in \omega_{f}(x)\right\}, \omega(f)=\bigcup\left\{\omega_{f}(x) ; x \in I\right\}, C(f)$ and $\Omega(f)$ denote the set of periodic points, the set of recurrent points, the set of $\omega$-limit points,

Received by the editors July 6, 1989 and, in revised form, October 16, 1989.

1980 Mathematics Subject Classification (1985 Revision). Primary 26A18, 58F08; Secondary 58F13, 28D15, 54H20. 
the center of $f$, and the set of nonwandering points of a map $f$, respectively. $A P(f)=\{x \in I$; for any neighborhood $U$ of $x$ there is a positive integer $n$ with $f^{n i}(x) \in U$ for $\left.i=0,1,2, \ldots\right\}$ is the set of almost periodic points of f. $A P B(f)=\{x \in I$; for any neighborhood $U$ of $x$ there is a $k>0$ such that for any $i$ there is a $j$ with $i<j \leq i+k$ and $\left.f^{j}(x) \in U\right\}$ is the set of points almost periodic in the sense of Bohr. $B(f)=\{x \in I$; for any $\epsilon>0$ there is a sequence $\left\{x_{i}\right\}_{i=0}^{n}$ with $x_{0}=x_{n}=x$ and $\left|f\left(x_{i}\right)-x_{i+1}\right|<\epsilon$ for any $i<n\}$ is the set of chain recurrent points of $f$. It is well known (cf., e.g. [10]) that for any $f \in C(I, I)$,

(3) $\operatorname{Per}(f) \subseteq A P(f) \subseteq A P B(f) \subseteq \operatorname{Rec}(f) \subseteq C(f) \subseteq \omega(f) \subseteq \Omega(f) \subseteq B(f)$.

An interval $J \subseteq I$ is periodic relative to a map $f$, if there is an $m>0$ with $f^{m}(J) \subseteq J$ and $\operatorname{int}(J) \cap \operatorname{int}\left(f^{i}(J)\right)=\varnothing$ for $i=1, \ldots, m-1$. The trajectory of a point $x$ is approximable by periodic trajectories, if for every $\epsilon>0$ there are $p \in \operatorname{Per}(f)$ and $n>0$ such that $\left|f^{i}(x)-f^{i}(p)\right|<\epsilon$ for every $i>n$. The trajectory of $x$ is approximable by trajectories of period intervals, if for any $\epsilon>0$ there are periodic interval $J$ and $n>0$ such that at least one interval in the orbit of $J$ has diameter less than $\epsilon$, and $\operatorname{dist}\left(f^{i}(x), f^{i}(J)\right)<\epsilon$ for any $i>n$.

We say that a closed invariant set $F$ of a map $f$ splits into two subsets $F_{0}, F_{1}$, if $F_{0}, F_{1}$ are nonempty sets contained in disjoint compact intervals with $F=F_{0} \cup F_{1}, f\left(F_{0}\right)=F_{1}$ and $f\left(F_{1}\right)=F_{0} ; F$ splits $k$-times, if $F$ splits into $F_{0}$ and $F_{1}$, and each $F_{i}, i=0,1$ splits $(k-1)$-times, under $f^{2}$. A compact invariant set $F$ of a map $f$ is said to be simple, if either $F$ is a periodic orbit of period $2^{k}$ for some $k \geq 0$, that splits $k$-times (cf. [2]), or if $F$ splits $k$-times for any $k>0$.

The paper is organized as follows: The next section contains main new results, along with Theorem $\mathrm{A}$ that contains related known results and will be used as a standard reference. In $\S 3$ we give preliminary constructions; some of then (Proposition 3.2), however, are interesting in themselves. The last section contains the proofs.

\section{MAIN RESUlts}

2.1. Theorem. For $f \in C(I, I)$, the following conditions are equivalent:

(i) $f$ is not chaotic;

(ii) $f \mid \omega(f)$ is stable in the sense of Ljapunov.

2.2. Remark. There are maps similar to a map from [14] that are stable on $C(f)$ and not on $\omega(f)$. Hence, in (ii) from Theorem 2.1, $\omega(f)$ cannot be replaced by a smaller set from (3). On the other hand, it is an open problem, whether in (ii), $\omega(f)$ can be replaced by $\Omega(f)$.

Combining Theorem 2.1 with known results, we can get the following result:

2.3. Theorem. For $f \in C(I, I)$, the following conditions are equivalent:

(i) $f$ is not chaotic;

(ii) $f \mid \omega(f)$ is stable in the sense of Ljapunov; 
(iii) $\omega(f)=\left\{x \in I ; \lim _{n \rightarrow \infty} f^{2^{n}}(x)=x\right\}$;

(iv) $A P(f)=\omega(f)$;

(v) every trajectory is approximable by periodic trajectories;

(vi) any two points belonging to arbitrary infinite $\omega_{f}(x)$ are contained in two disjoint periodic intervals.

Proof. The equivalence of conditions (i), (v), and (vi) is proved in [12, 4], equivalence of (i) and (ii) is given in Theorem 2.1, and equivalence of (ii), (iii), and (iv) in [11] (cf. also [10]).

2.4. Theorem. Let $f \in C(I, I)$. The following conditions are equivalent:

(i) $h(f)=0$;

(ii) $h(f \mid \operatorname{Per}(f))=0$;

(iii) $f \mid A P B(f)$ is not chaotic, i.e. for any minimal set $M, f \mid M$ is not chaotic;

(iv) $f \mid B(f)$ is not chaotic;

(v) every trajectory is approximable by trajectories of periodic intervals.

The above theorem gives alternative characterizations of the class of maps with zero topological entropy. For completeness, we present here also the known results as Theorem $\mathrm{A}$, and will use them in the sequel as a standard reference.

Theorem A. Let $f \in C(I, I)$. The following conditions are equivalent:

(i) $h(f)=0$;

(ii) periodic of every periodic orbit is a power of 2 ;

(iii) every periodic orbit is simple;

(iv) there are no homoclinic trajectories;

(v) $\operatorname{Per}(f)$ is a $G_{\delta}$ set;

(vi) $A P B(f)$ is a $F_{\sigma}$ set;

(vii) $f \mid \operatorname{Per}(f)$ is stable in the sense of Ljapunov;

(viii) $\operatorname{Rec}(f)=A P B(f)$, i.e. every closed transitive and invariant set is minimal;

(ix) $A P(f)=\left\{x \in I ; \lim _{n \rightarrow \infty} f^{2^{n}}(x)=x\right\}$;

(x) every $\omega$-limit set is simple;

(xi) every $\omega$-limit set contains a unique minimal set;

(xii) if $\omega_{f}(x)$ is infinite then $\omega_{f}(x) \cap \operatorname{Per}(f)=\varnothing$;

(xiii) if $J_{0}, J_{1}$ are intervals having at most one point in common, then for any $m, J_{0} \cup J_{1}$ is not contained in $f^{m}\left(J_{0}\right) \cap f^{m}\left(J_{1}\right)$, i.e. $f$ has no horseshoe;

(xiv) if $F$ is an invariant set and $m$ a positive integer, then $f^{m} \mid F$ is not topologically conjugated to the shift $\tau$ on the space of sequences of two symbols 0 and 1 ;

(xv) for any $\omega$-limit set $F, A(F)=\left\{x ; \omega_{f}(x)=F\right\}$ is a set of the 2 nd Baire class;

(xvi) $B(f)$ is the union of all simple sets in $I$. 
The results from Theorem A can be found in [11] (cf. also [10]), including complete list of references.

\section{Preliminary Results}

3.1. Lemma. A closed invariant set $M$ of a map $f \in C(I, I)$ splits $n$-times iff for every $k \leq n$ there is a decomposition $\left\{M(i, k) ; i=1, \ldots, 2^{k}\right\}$ of $M$ such that any two sets $M(i, k), M(j, k)$, are separated by disjoint compact intervals if $i \neq j$, and $f(M(i, k))=M(i+1, k)$ for any $i\left(\bmod 2^{k}\right)$.

The proof is easy and we omit it.

Any decomposition from Lemma 3.1 is called a cyclic decomposition of $M$ of degree $k$. It is easy to see that such a cyclic decomposition is unique.

It is known (cf., eg. [1] or [12]) that if $h(f)=0$ then every infinite $\omega_{f}(x)$ is contained in a set of the form $\bigcap_{k=0}^{\infty} \bigcup\left\{f^{i}\left(J_{k}\right) ; i=1,2, \ldots, 2^{k}\right\}$, where every $J_{k}$ is a compact periodic interval of period $2^{k}$, satisfying $J_{k} \supset J_{k+1}$ for each $k$. In the sequel, however, we need the intervals $J_{k}$ along with their images $f^{i}\left(J_{k}\right)$ to be open. Therefore we prove the following proposition.

3.2. Proposition. Let $f \in C(I, I), h(f)=0$ and let $\omega_{f}(x)$ be infinite. Let $\left\{M(i, k) ; i=1, \ldots, 2^{k}\right\}$ be a cyclic decomposition of $\omega_{f}(x)$ of degree $k$. Then there are intervals $J(i, k), i=1, \ldots, 2^{k}$, relatively open in $I$ and such that for any $i, j, i \neq j$,

(i) $\overline{J(i, k)} \cap \overline{J(j, k)}=\varnothing$;

(ii) $M(i, k) \subset J(i, k)$;

(iii) $f^{2^{n}}(\overline{J(i, k)}) \subseteq J(i, k)$.

Proof is based on the following two lemmas.

3.3. Lemma. Let $f \in C(I, I)$ with $h(f)=0$, and let $f(y) \leq x<y \leq f(x)$, for some $x, y$. Then there are no fixed points of $f$ in $[f(y), x] \cup[y, f(x)]$.

Proof. See [7] or note that the result follows easily from (xiii) in Theorem A.

3.4. Lemma. Let $f \in C(I, I), h(f)=0$ and let $\omega_{f}(x)$ be infinite. Let $U=[u, v]$ be the convex hull of $\omega_{f}(x)$ and $V=[a, b]$ the minimal compact invariant interval containing $U$. Then

(i) $V \backslash U$ contains no fixed point of $f$;

(ii) there is an interval $J$ relatively open in I such that $U \subseteq J, J \backslash U$ contains no fixed points of $f$, and $f(\bar{J}) \subseteq J$.

Proof. (i) Assume, e.g. that $p$ is the largest fixed point of $f$ in $[a, u]$. By (xii) in Theorem A, $f(u)>u$, hence for any $y \in(p, u], f(y)>y$, and since $V$ is minimal, there is the least $q>u, q \in V$, with $f(q)=p$. Now

$$
p<f^{n}(y)<q \text { for any } y \in(p, q) \text { and } n=0,1,2, \ldots
$$


since otherwise there would be some $z$ with $f(q) \leq p(=f(p))<z<q \leq f(z)$, contrary to Lemma 3.3. But (4) and $f(q)=p<q$ imply that

$$
\max \{f(y) ; y \in(p, q)\}=\max \{f(y) ; y \in[p, q]\}=r<q,
$$

and clearly $v \leq r$. By the minimality of $q, \min \{f(y) ; y \in[u, r]\}=s>p$ and since $\omega_{f}(x)$ is invariant, there is $s \leq u$. Thus $K=[s, r]$ is an invariant interval properly contained in $V$ and containing $\omega_{f}(x)$ - a contradiction.

(ii) Let $W=[c, d]$ be the maximal interval such that $U \subseteq W$ and $\operatorname{int}(W \backslash U)$ contains no fixed point of $f$. If the endpoints of $W$ are not fixed, then $W=I$, and clearly $J=I$ is the desired interval.

So assume that, e.g. $f(c)=c$. By (i), $c<a<b \leq d$. Let $r$ be the minimal point from $[b, d]$ such that, for any $y \in[b, d], f(r) \leq \max \{c, f(y)\}$. Denote $\alpha=\min \{a, f(r)\} \geq c$. Let $s \in[\alpha, a]$ be such that $f(y) \leq f(s)$ for $y \in[\alpha, a]$. Denote $\beta=\max \{f(s), b\}$. Consider the following two cases. Case A. $\alpha>c$. Assume

$$
f(\beta)<\beta \leq d .
$$

Then there is a right-hand neighborhood $H$ of $\beta$, relatively open in $I$ (if $\beta$ is not the end-point of $I$, take $H \neq \varnothing$ ) such that $f(H) \subseteq[\alpha, \beta]$. Since $\alpha<f(\alpha) \leq \beta$, there is an open left-hand neighborhood $G$ of $\alpha$ (nonempty, if $I$ is not the endpoint of $I$ ) such that $f(\bar{G}) \subseteq[\alpha, \beta] \cup H$. For $J=G \cup[\alpha, \beta] \cup H$ we have $f(\bar{J})=f(\bar{G}) \cup f([\alpha, \beta]) \cup f(\bar{H}) \subseteq J$.

It remains to prove (5). If $f(d)<d$ then (5) is clearly true. So let $f(d)=d$. It suffices to show that $\beta<d$. Assume the contrary. Then for $J_{0}=[s, a]$, $J_{1}=[a, d], f^{2}\left(J_{0}\right) \cap f^{2}\left(J_{1}\right) \supseteq f([b, d]) \supseteq[\alpha, \beta] \supseteq J_{0} \cup J_{1}$, contrary to (xiii) in Theorem A.

Case B. $\alpha=c$. Then $\beta<r$, since otherwise, for $J_{0}=[c, a], J_{1}=[s, a]$, we have $f^{2}\left(J_{0}\right) \cap f^{2}\left(J_{1}\right) \supseteq J_{0} \cup J_{1}$-a contradiction. Denote $\gamma=\min \{f(y) ; y \in$ $[a, \beta]\}$. By the minimality of $r, c<\gamma$ and clearly $f(\gamma)>\gamma$. So there is an open neighborhood $G$ of $\gamma$ with $f(\bar{G}) \subseteq[\gamma, \beta]$, and an open neighborhood $H$ of $\beta$ with $f(\bar{H}) \subseteq G \cup[\alpha, \beta]$. But then $J=G \cup[\alpha, \beta] \cup H$ is the desired interval.

Proof of Proposition 3.2. Let $g=f^{2^{k}}$. The sets $M(i, k)=\omega_{g}\left(f^{i}(x)\right)$ are closely $\omega$-limit sets of $g$, separated by fixed points of $g$. Thus we can apply (ii) in Lemma 3.4 to give sets $J(i, k)$ satisfying all conditions.

3.5. Lemma. Let $M$ be a simple invariant set of a map $f \in C(I, I)$. Then $f \mid M$ is not chaotic.

Proof. Let $x, y \in M, x \neq y$. If $M$ is a cycle, then (2) is not there. Thus let $M$ be an infinite simple set. By Lemma 3.1, for any $k$ there is a cyclic decomposition $\left\{M(i, k) ; i=1, \ldots, 2^{k}\right\}$ of $M$. If for some $k, x$ and $y$ belong to disjoint sets $M(i, k)$ and $M(j, k)$, then again (2) is not satisfied.

So we can assume that for any $k$ there is some $i$ with $x, y \in M(i, k)$; without loss of generality we can assume that $x, y \in M(1, k)$ for each $k$. Let 
(1) be true. Then there are an $\epsilon>0$ and an increasing sequence $\{n(i)\}_{i=1}^{\infty}$ of positive integers such that $\left|f^{n(i)}(x)-f^{n(i)}(y)\right|>\epsilon$ for every $i$. For any $j$, let $k=k(j)$ be such that $2^{k}>n(j)$. Then $\left\{f^{n(i)}(x), f^{n(i)}(y)\right\} \subset$ $M(1+n(i), k)$, and the sets $M(1+n(i), k)$ are pairwise disjoint for $i=1$, $\ldots, j$. Hence, $\operatorname{diam} M \geq \sum_{i=1}^{j} \operatorname{diam} M(1+n(i), k)>j \epsilon$, for any $j$, and this is a contradiction.

3.6. Lemma. Let $f \in C(I, I)$ and $A \subseteq I, f(A) \subseteq A$. Then $h(f \mid A)=$ $h(f \mid \bar{A})$.

Proof. This lemma must be known but we are not able to give a reference. For simplicity put $A=A_{1}$ and $\bar{A}=A_{2}$. For a given $\epsilon>0, i \in\{1,2\}$ and a positive integer $n$, let $S_{i}(f, \epsilon, n) \subset A_{1}$ be a maximal set such that for any $x, y \in S_{i}(f, \epsilon, n), x \neq y$, there is an integer $k$ with $0 \leq k<n$ and $\left|f^{k}(x)-f^{k}(y)\right|>\epsilon$. Since

$$
h\left(f \mid A_{i}\right)=\lim _{\epsilon \rightarrow 0} \limsup _{n \rightarrow \infty} \frac{1}{n} \log \operatorname{card}\left(S_{i}(f, \epsilon, n)\right)
$$

(cf. [3]), it suffices to show that for any $\epsilon>0$,

$$
\operatorname{card}\left(S_{1}(f, \epsilon / 3, n) \geq \operatorname{card}\left(S_{2}(f, \epsilon, n)\right)\right.
$$

since the inequality $h\left(f \mid A_{1}\right) \leq h\left(f \mid A_{2}\right)$ is trivial. Thus let $x_{1}, \ldots, x_{m}$ be the elements of $S_{2}(f, \epsilon, n)$. Since $\bar{A}_{1}=A_{2}$, by the continuity of $f$ there are points $y_{1}, \ldots, y_{m}$ from $A_{1}$ such that for any $j \in\{1, \ldots, m\}$ and any $r \in\{0, \ldots, n\},\left|f^{r}\left(x_{j}\right)-f^{r}\left(y_{j}\right)\right|<\epsilon / 3$. Now if for some $k \in\{0, \ldots, n-1\}$ we have $\left|f^{k}\left(x_{i}\right)-f^{k}\left(x_{j}\right)\right|>\epsilon$, then clearly $\left|f^{k}\left(y_{i}\right)-f^{k}\left(y_{j}\right)\right|>\epsilon / 3$. Thus $\left\{y_{1}, \ldots, y_{m}\right\}$ is contained in a maximal set $S_{1}(f, \epsilon / 3, n)$ and (6) follows.

\section{Proofs of MAIN Results}

Proof of Theorem 2.1 . (i) $\Rightarrow$ (ii). Let $f$ be nonchaotic. Then by [12], $h(f)=$ 0 , by $(x)$ in Theorem A, every $\omega$-limit set is simple, and again by [12] (or by (vi) in Theorem 2.3), every two points of any infinite $\omega$-limit set are separable by disjoint periodic intervals.

Let $a \in \operatorname{Per}(f)$. We prove that $f \mid \omega(f)$ is stable at $a$. Without loss of generality we may assume that $f(a)=a$. Let $\epsilon_{0}>0$. Choose numbers $\left\{\epsilon_{i}\right\}_{i=1}^{5}$ such that for every $i>0, \epsilon_{i-1}>\epsilon_{i}>0$ and $|u-v|<\epsilon_{i}, u, v \in I$ imply $|f(u)-f(v)|<\epsilon_{i-1}$. Let $b \in\left(a-\epsilon_{5}, a+\epsilon_{5}\right) \cap \omega(f)$. If $f^{2}(b)=b$ then $\left|f^{i}(b)-a\right|<\epsilon_{0}$ for every $i$. Otherwise $b$ belongs to a simple set $M$, that splits 2-times. Let $\{M(i, 2) ; i=1, \ldots, 4\}$ be the corresponding decomposition of $M$. Then it is easy to see that at least one of the sets $M(i, 2)$ lies in $\left(a-\epsilon_{3}, a+\epsilon_{3}\right)$, and consequently, $M \subset\left(a-\epsilon_{0}, a+\epsilon_{0}\right)$, i.e. $\left|f^{i}(b)-a\right|<\epsilon_{0}$ for every $i$.

Now let $a \in \omega(f) \backslash \operatorname{Per}(f)$. Denote by $M$ an $\omega$-limit set containing $a$. Let $J(i, k)$ be open intervals as in Proposition 3.2. Since every two points from 
$M$ are separated by periodic intervals, for every $\epsilon>0$ there is a $k=k(\epsilon)$ such that $\operatorname{diam}(J(i, k) \cap \omega(f))<\epsilon$ for every $i=1, \ldots, 2^{k}$. Now for some $j, J(j, k)$ is a neighborhood of $a$, and this implies the stability of $f \mid \omega(f)$ at $a$.

(ii) $\Rightarrow$ (i). Let $f \mid \omega(f)$ be stable. Then also $f \mid \operatorname{Per}(f)$ is stable in the sense of Ljapunov and by Theorem A, every $\omega$-limit set is simple. Let $M$ be an infinite $\omega$-limit set, and let $u, v \in M,|u-v|=\epsilon>0$. Let for each $k,\left\{M(i, k) ; i=1, \ldots, 2^{k}\right\}$ be the cyclic decomposition of $M$ of degree $k$. Since $f \mid M$ is stable, there is a $k$ such that $\operatorname{diam}(M(i, k))<\epsilon$ for every $i=1, \ldots, 2^{k}$. Hence $u$ and $v$ belong to different components $M(i, k)$, $M(j, k)$, and so, by Proposition 3.2, to disjoint periodic intervals $J(i, k)$, $J(j, k)$. By [12], $f$ is not chaotic.

Proof of Theorem 2.4. (i) $\Rightarrow$ (ii). By [3], $h(f)=h(f \mid C(f))$, and by [8], $C(f)=\overline{\operatorname{Per}(f)}$. Now apply Lemma 3.6.

(i) $\Rightarrow$ (iv). Let $h(f)=0$. Let $x, y \in B(f), x \neq y$. By (xvi) in Theorem A there are maximal simple sets $M(x)$ and $M(y)$, containing $x$ and $y$, respectively. If $M(x)=M(y)$, the result follows from Lemma 3.5. If $M(x) \neq M(y)$, then we can easily see that $M(x) \cap M(y)=\varnothing$ and since both $M(x), M(y)$ are compact, (2) is not true.

(iv) $\Rightarrow$ (iii). Trivially, $f \mid A P B(f)$ is not chaotic. The rest follows from the Birkhoff's theorem on minimal sets: every minimal set of $f$ is contained in $A P B(f)$, and conversely, for any $x \in A P B(f), \overline{\operatorname{Orb}(x)}$ is a minimal set.

(iii) $\Rightarrow$ (i). Assume $h(f)>0$. We show that $f \mid A P B(f)$ is chaotic. By Theorem A there is an invariant set $F \subset I$, and an integer $m$ such that $f^{m} \mid F$ is topologically conjugated to the shift $\tau$ on the space $\{0,1\}^{N}$ of sequences of two symbols 0 and 1 . It suffices to show that $\tau \mid A P B(\tau)$ is chaotic.

For any nonnegative integer $n$ define blocks $A_{n}, B_{n}$ of length $4^{n}$, consisting of symbols from $\{0,1\}$ as follows: $A_{0}=0, B_{0}=1$,

$$
A_{k+1}=A_{k} A_{k} B_{k} A_{k} \quad \text { and } \quad B_{k+1}=B_{k} A_{k} B_{k} B_{k}
$$

for $k \geq 0$. Let $\alpha, \beta \in\{0,1\}^{N}$ be such that, for any $k$, the first $4^{k}$ digits of $\alpha$ and $\beta$ coincide with the digits of $A_{k}$ and $B_{k}$; respectively. It is easy to see that, for any $k, \alpha$ and $\beta$ are in $\left\{A_{k}, B_{k}\right\}^{N}$ and both $\alpha$ and $\beta$ contain no substrings of the form $A_{k} A_{k} A_{k} A_{k}$ or $B_{k} B_{k} B_{k} B_{k}$. Hence, for every $i$ there is an $n$ with $i \leq n<i+4^{k+1}$ such that $\tau^{n}(\alpha)$ begins with $A_{k}$. Since $\alpha$ begins with $A_{k}$, too, we have $\alpha \in A P B(\tau)$. Similarly, $\beta \in A P B(\tau)$.

If $m=4^{n}$ then both $\tau^{m}(\alpha), \tau^{m}(\beta)$ begin with $A_{n}$ (see (7)), hence

$$
\liminf _{k \rightarrow \infty} \rho\left(\tau^{k}(\alpha), \tau^{k}(\beta)\right)=0,
$$

where $\rho$ is a metric of pointwise convergence in $\{0,1\}^{N}$. Since clearly, for infinitely many $k, \tau^{k}(\alpha)$ begins with 0 and $\tau^{k}(\beta)$ with 1 , we have

$$
\limsup _{k \rightarrow \infty} \rho\left(\tau^{k}(\alpha), \tau^{k}(\beta)\right)>0 .
$$


(i) $\Rightarrow(\mathrm{v})$. Let $h(f)=0$ and $x \in I$. If $\omega_{f}(x)$ is cycle, then the trajectory of $x$ is a trajectory of degenerated periodic interval $J=\{x\}$. So let $\omega_{f}(x)$ be infinite. For each $k$, let $\left\{J(i, k) ; i=1, \ldots, 2^{k}\right\}$ be the intervals from Proposition 3.2. We may assume that $x \in J(1, k)$, for each $k$. Since $J(1, k) \supset J(1, a)$ whenever $s>k$, for every $\epsilon>0$ there are $k$ and $i$ with $1 \leq i \leq 2^{k}$ such that $\operatorname{diam}(J(i, k))<\epsilon$. This implies $(\mathrm{v})$.

$(\mathrm{v}) \Rightarrow(\mathrm{i})$. Let $h(f)>0$. We show that then the trajectory of a point $x$ is approximable by trajectory of no periodic interval. Clearly, we can take as $x$ any point such that $\omega_{f}(x)$ is the closure of a homoclinic trajectory. By Theorem $\mathrm{A}$ it suffices to show that the shift $\tau$ on $\{0,1\}^{N}$ has this property. It is easy to verify that we can take $x=A_{1} A_{2} A_{3} \ldots$, where $A_{i}=100 \ldots 0$ (a single " 1 " followed by $i$ zeros).

\section{REFERENCES}

1. A. M. Blokh, On limit behavior of one-dimensional dynamical systems, Uspekhi Mat. Nauk 37 (1982), 137-138. (Russian)

2. L. Block, Simple periodic orbits of mappings of the interval, Trans. Amer. Math. Soc. 254 (1979), 391-398.

3. R. Bowen, Topological entropy and Axiom A, Global Analysis, Proc. Sympos. Pure Math. 14 (1970), 23-41.

4. K. Janková and J. Smital, A characterization of chaos, Bull. Austra!. Math. Soc. 34 (1986), 283-292.

5. M. Kuchta and J. Smital, Two point scrambled set implies chaos, Proc. Europ. Conf. on Iteration Theory (Caldas de Malavella, Spain, 1987), World Scientific, 1989, 427-430.

6. M. Misiurewicz and J. Smital, Smooth chaotic maps with zero topological entropy, Ergodic Theory Dynamical Systems 8 (1988), 421-424.

7. D. Preiss and J. Smital, A characterization of non-chaotic maps of the interval stable under small perturbations, Trans. Amer. Math. Soc. (to appear).

8. A. N. Sarkovskii, Nonwandering points and the center of a continuous mapping of the line into itself, Dopovidi Akad. Nauk Ukraiin RSR Ser. A. No. 7 (1964), 865-868. (Ukrainian)

9. ㄴ The partially ordered system of attracting sets, Soviet Math. Dokl. 7 (1966), 13841386.

10. A. N. Šarkovskii, J. L. Majstrenko, and B. J. Romanenko, Difference equations and their applications, Naukova Dumka, Kiev, 1986. (Russian)

11. A. N. Šarkovskii, S. F. Koljada, A. G. Sivak, and V. V. Fedorenko, Dynamics of onedimensional mappings, Naukova Dumka, Kiev, 1989. (Russian)

12. J. Smital, Chaotic maps with zero topological entropy, Trans. Amer. Math. Soc. 297 (1986), 269-282.

13. J. Xiong, Set of almost periodic points of a continuous self-map of an interval, Acta. Math. Sinica (N.S.) 2 (1986), 73-77.

14. M. B. Verejkina and A. N. Šarkovskii, Recurrence in one-dimensional dynamical systems, Approx. and Qualitative Methods of the Theory of Differential-Functional Equations, Inst. Math. Akad. Nauk USSR, Kiev, 1983, pp. 35-46. (Russian)

(V. V. Fedorenko and A. N. Šarkovskii) Institute of Mathematics, Ukrainian Academy of SCIENCES, REPINA 3, KIEV, USSR

(J. Smital) Institute of Mathematics, Comenius University, 84215 Bratislava, Czecho SLOVAKIA 